\title{
Structure-assisted design of inhibitors of CA IX enzyme based on polyhedral boron compounds
}

\author{
Jiří Brynda, ${ }^{1,4}$ Michael Kugler, ${ }^{1,4}$ Jan Nekvinda, ${ }^{2}$ Josef Holub, ${ }^{2}$ Suzan El Anwar, ${ }^{2}$ Viswanath Das, ${ }^{3}$ Václav Šícha, ${ }^{2}$ \\ Klára Pospíšilová, ${ }^{1}$ Milan Fábry ${ }^{4}$, Vlastimil Král, ${ }^{4}$ Petr Pachl, ${ }^{1}$ Marián Hajdúch, ${ }^{3}$ Bohumír Grüner ${ }^{2}$, \\ Pavlína Řezáčová, ${ }^{1,4}$ \\ ${ }^{1}$ Institute of Organic Chemistry and Biochemistry of the Czech Academy of Sciences \\ Flemingovo nám. 2, 16610 Prague, Czech Republic \\ ${ }^{2}$ Institute of Inorganic Chemistry of the Czech Academy of Sciences, Hlavni 1001, 25068 Řez, Czech Republic \\ ${ }^{3}$ Institute of Molecular and Translational Medicine, Hněvotinská 1333/5, 77900 Olomouc, Czech Republic \\ ${ }^{4}$ Institute of Molecular Genetics of the Czech Academy of Sciences, Videňská 1083, 14220 Prague, Czech Republic \\ brynda@uochb.cas.cz
}

This poster describes structure-assisted design of inhibitors of human carbonic anhydrase IX (CA IX) based on three-dimensional carborane and cobalt bis(dicarbollide) clusters. CA IX enzyme is known to play crucial role in cancer cell proliferation and formation of metastases. The new class of potent and selective CA IX inhibitors combines structural motif of bulky inorganic cluster with an alkylsulfamido or alkylsulfonamido anchor group for $\mathrm{Zn}^{2+}$ atom in the enzyme active site. Detailed structure-activity relationship (SAR) study of a large series containing 50 compounds is corroborated by almost 50 high resolution structures of compounds bound to CA IX active site and the active site of CA II. Structural features of the cluster-containing inhibitors that important for efficient and selective inhibition of CA IX activity were uncovered and used in structure-assisted design. Preclinical evaluation of selected compounds revealed low toxicity, favourable pharmacokinetics and ability to reduce tumour growth. Cluster-containing inhibitors of CA IX can thus be considered as promising candidates for drug development and/ or for combination therapy in boron neutron capture therapy.

Keywords: carborane, cobal bis(dicarbollide), carbonic anhydrase, enzyme inhibition, SAR study 